\title{
Droit positif et sacré : l'exemple du droit de propriété inspiré de la Déclaration des droits de l'homme et du citoyen
}

Positive and sacred law: the example of the right to property inspired by the Declaration of the Rights of Man and of the Citizen

\section{Pierre-Yves Chicot}

\section{(2) OpenEdition Journals}

\section{Édition électronique}

URL : http://journals.openedition.org/add/709

DOI : $10.4000 /$ add.709

ISSN : 2606-1988

\section{Éditeur}

Presses universitaires de Rouen et du Havre

\section{Édition imprimée}

Date de publication : 1 mai 2014

Pagination : 33-57

ISBN : 979-10-240-0161-6

ISSN : 1955-0855

\section{Référence électronique}

Pierre-Yves Chicot, «Droit positif et sacré : l'exemple du droit de propriété inspiré de la Déclaration des droits de l'homme et du citoyen », Les Annales de droit [En ligne], 8 | 2014, mis en ligne le 14 mars 2018, consulté le 17 septembre 2019. URL : http://journals.openedition.org/add/709; DOI : 10.4000/add. 709 


\title{
Droit positif et sacré : l'exemple du droit de propriété inspiré de la Déclaration des droits de l'homme et du citoyen
}

\author{
Pierre-Yves CHIcot
}

Tel qu'il se trouve consigné dans les Écritures, le normativisme divin détermine les comportements collectifs et individuels. Il suffit pour s'en rendre compte d'examiner les codes de régulation sociale dont certaines dispositions trouvent leur origine dans la morale originelle. Par exemple, dans les pays de confession chrétienne le droit pénal puise par endroit sa source dans le décalogue ${ }^{1}$.

Figurant parmi les dix commandements, l'injonction comminatoire "tu ne tueras point», emporte pour conséquence juridique que le meurtre soit soumis à un régime de coercition prévu par des textes de droit positif introduits dans le Code pénal. Autre exemple, en droit administratif, la moralité ${ }^{2}$ et le respect de la dignité de la personne humaine ${ }^{3}$ sont des composantes structurantes de la notion d'ordre public. Les notions de moralité et de dignité qui sont prises en compte aussi bien par le droit public que par le droit privé revêtent pour les sciences juridiques une importance fondamentale. La notion de dignité procède d'une démarche empreinte de morale ${ }^{4}$. Elle constitue avec la justice, un principe premier saisi par le droit. Que ce soit d'un point de vue laïc ou théocratique, même la personne morte, nous précise Muriel Fabre-Magnan, est titulaire de droits au nom de la dignité 5

1. On pourrait rapprocher la charia islamique du décalogue chrétien. Voir Julien Loiseau, "Qu'est-ce que la charia?», Le Débat, n 171, 2012/4, p. 172-178.

2. CE, 25 janvier 1924, Chambre syndicale de la cinématographie, Recueil Lebon, p. 94. Le Conseil d'État dans cet arrêt invoque «les bonnes mœurs» et «l'influence pernicieuse sur la moralité publique » à propos de la diffusion d'un film. Voir également CE, 8 décembre 1997, Commune d'Arcueil, Recueil Lebon, p. 482 ; et CE, 4 février 2004, Association Promouvoir, Recueil Lebon. t. 887-888.

3. CE, 27 octobre 1995, Commune de Morsang-sur-Orge, Recueil Lebon, p. 372.

4. Voir Thomas de Koninck et Gilbert Larochelle, La Dignité humaine, Paris, PUF, 2005.

5. Voir Muriel Fabre-Magnan, Introduction au droit, Paris, PUF, « Que sais-je ?», 2010. 
Le droit positif et le sacré, loin d'entretenir une relation de distance, se côtoient allègrement. Le premier étant fréquemment nourri par le second. Il est intéressant d'analyser le maillage entre droit positif et sacré, en prenant pour base d'appui le droit de propriété. La Déclaration des droits de l'homme et du citoyen du 26 août 1789 dispose que «la propriété est un droit inviolable et sacré, nul ne peut en être privé ». Est-il possible de retrouver dans le droit chrétien d'origine divine une telle présentation du droit de propriété?

Qu'on y réponde par oui comme c'est le cas pour certains auteurs ${ }^{6}$, ou par non ${ }^{7}$, l'essentiel ici consiste à mettre en lumière la finalité de l'invocation au sacré. En effet, il semble bien que l'objectif du pouvoir normatif d'obédience laïque est d'utiliser la force de la normativité du sacré pour renforcer l'effet contraignant de la disposition visée. Il s'agit donc de convaincre les destinataires de la norme du bien-fondé de la parole de droit positif énoncée et de l'obligation supérieure qui existe à la respecter en invoquant précisément le sacré.

La propriété peut relever soit du régime collectif ou public, soit du régime individuel. En se référant au texte fondateur du 26 août 1789 , la haute protection dont il est fait mention concerne le droit de propriété privée. Le pouvoir constituant, en revêtant le droit de propriété privée d'une onction sacrée, manifeste la volonté de lui donner un caractère éminemment solennel, afin d'en garantir la protection pour des temps immémoriaux. C'est l'allusion même indirecte au champ du divin qui doit concourir à la portée indélébile de la disposition.

Dans le même ordre d'idée, on fera observer qu'il n'est pas rare d'entendre les juristes parler de «sacro-saint principe ${ }^{8}$ » pour montrer l'emprise inextinguible de celui-ci sur les sujets de droit. Et ce, quand bien même le droit divin le méconnaîtrait. On pourrait citer à titre d'exemple «le sacro-saint principe d'égalité » qui témoigne de «l'impératif égalitaire" au fondement de la République ${ }^{9}$. En ayant recours à la mention «sacro-saint principe», l'invocation du sacré joue comme

6. Marie-France Renoux-Zagamé, Origines théologiques du concept moderne de propriété, Genève, Librairie Droz, 1987.

7. Bernard Van Meenen, "Propriété ou appropriation? Regard sur les sources bibliques ", mars 2009, disponible sur http://paxchristiwb.be/publications/analyses/bible-eglises-propriete, o0oo151.html (consulté le 25 novembre 2013).

8. "Qui est à la fois saint et sacré. Qui a un caractère d'inviolabilité, auquel on ne peut déroger ", Centre national de ressources textuelles et lexicales, CNRS. Définition disponible en ligne à l'adresse http://www.cnrtl.fr/definition/sacro-saint (consulté le 25 novembre 2013). 
la marque d'une contrainte supérieure. Pour enjoindre à la plus grande observance de la règle, il y a la recherche d'un effet à double détente. Un premier effet qui va naître du caractère obligatoire du droit positif lui-même. Un second effet qui va relever de la force évocatrice de la dimension théologique sur l'inconscient individuel et collectif, faisant ainsi fonction d'opinio juris renforcé.

La place réservée au droit de propriété dans la taxonomie des droits dévolus aux citoyens révèle en même temps l'empreinte libérale de la Révolution française. Cet aspect des choses livre un nouveau motif d'intérêt pour ce sujet qui se propose, d'analyser également, le rapport entretenu entre l'approche économique d'un fait social temporel et son rattachement à la sphère intemporelle.

Dans le contexte de la Révolution française, le droit de propriété, énoncé comme "un droit inviolable et sacré", introduit l'idée sousjacente de la liberté individuelle, d'acquérir et de conserver ce qui a été acquis. Diderot reprenant à son compte le propos de Thomas Hobbes ${ }^{10}$ dira que le désir d'acquérir est une inclination générale, que partagent tous les hommes ${ }^{11}$. La liberté individuelle d'acquérir aura pour effet de rendre plus aisée l'effectivité de cette inclination générale. Relier droit de propriété et liberté individuelle contribue à former le socle du système économique libéral, dont la démonstration du triomphe n'est plus à faire en ce début de $\mathrm{xxI}^{\mathrm{e}}$ siècle.

Qu'on s'en réjouisse ou qu'on le déplore, ce succès à l'échelle mondiale témoigne de la sacralisation renouvelée du droit de propriété, équivalant à une sorte de sacre globalisé du régime de la propriété privée des moyens de production. Véhiculé par toutes les grandes institutions économiques et financières internationales (l'Organisation mondiale du commerce, le Fonds monétaire international, la Banque mondiale, la conférence des Nations unies pour le commerce et le développement, notamment) comme la pierre angulaire de la croissance et du développement économique, voire du bonheur des hommes, le libéralisme économique est consacré partout dans le monde ${ }^{12}$. Et ce en dépit de visions philosophiques et sociologiques qui récusent l'obsession quantitative.

9. Voir par exemple "Aux sources du Républicanisme français", dans Nouvelles fondations, $\mathrm{n}^{\mathrm{o}}$ 2, 2006/2, p. 173-176; et Jean-Fabien Spitz, Les Moments républicains en France, Paris, Gallimard, «NRF-Essais », 2005.

10. Thomas Hobbes, Leviathan, trad. François Tricaud, Paris, Sirey, 1971, p. 95.

11. Voir Florent Guénard, "La liberté et l'ordre public: Diderot et la bonté des lois ", dans Revue de Métaphysique et de Morale, $\mathrm{n}^{\circ}$ 45, 2005/1, p. 114 et suiv.

12. Voir Monique Chemiller-Gendreau, "L'apparent triomphe du libéralisme ou le triomphe de l'idée libérale?", L’homme et la société, nº 97, 1990, p. 39-43. 
Dans le même temps, si la puissance publique n'est pas affaiblie dans sa capacité à enserrer par des dispositions d'ordre public le droit de propriété privée, il est tout de même permis d'observer un éclatement de la notion de déréglementation ${ }^{13}$ qui bat en brèche une approche qui privilégierait la propriété publique des moyens de production. Ainsi progressivement, mais de manière résolue, c'est la conception du droit de propriété, qui se doit d'être privée qui tend à l'emporter ${ }^{14}$. Cette conception est tout particulièrement défendue avec opiniâtreté par ceux qui sont parfois désignés comme des "évangélistes du marché ${ }^{15}$ ". En l'occurrence, la référence au vocabulaire biblique est une nouvelle fois symptomatique du lien que peut entretenir la sphère économique d'essence terrestre et la sphère religieuse d'essence céleste.

De la Révolution française libérale au phénomène plus récent de la globalisation des marchés, la convergence est cristallisée par un état d'esprit qui consiste en la louange de la propriété privée et de la liberté individuelle, comme éléments fondamentaux et structurants de l'ordre social. Aussi, peut-on observer une sacralisation de la propriété privée comme relevant d'un ordre ancien (1) renouvelé, conduisant à la sacralisation moderne du régime de la propriété privée des moyens de production (2).

\section{De la sacralisation ancienne de la propriété privée comme fondement de l'ordre social révolutionnaire}

L'homme, quelle que soit sa civilisation ou sa confession est sujet à l'invocation d'une forme de transcendance d'essence matérielle ou immatérielle. Le recours à la Nature ou à Dieu traduit une forme de quête

13. Renan Le Mestre, La soumission des secteurs publics nationaux à la déréglementation communautaire, thèse de doctorat en droit, faculté de droit et des sciences politiques, Nantes, 1998, 517 p. ; Louis Dubouis, «La déréglementation et le droit communautaire », RFDA, 1986, p. 483-493; Alberto Pera, «La déréglementation et la privatisation dans une perspective macroéconomique ", Revue économique de l'OCDE, disponible sur http://www.oecd.org/dataoecd/18/44/35381793.pdf (consulté le 24 octobre 2013); Robert Boyer, Mario Dehove et Dominique Plihon, Les Crises financières, Paris, La Documentation française, «Les Rapports du Conseil d'analyse économique », 2004.

14. Voir Nicolas Thirion, «Privatisations d'entreprises publiques, économie de marché et transformation des systèmes juridiques étatiques: un processus inéluctable? ", Pyramides, revue du Centre de recherche en administration publique, 2000/2, p. 85-110.

15. Keith Dixon, Les Évangélistes du marché: les intellectuels britanniques et le néolibéralisme, Paris, Éd. Raisons d'agir, « Raisons d'agir », 1998. 
du Sacré ${ }^{16}$ pouvant être défini comme ce qui est au-dessus de lui, ou ce qui l'a précédé, ou les deux à la fois.

Évidemment, bien des choses relèvent de la création humaine. Mais la création n'est-elle pas toujours inspirée, symbolisant à l'envie que l'esprit domine la matière? Il en va ainsi de la création normative. L'origine du droit positif pouvant être, à bien des égards, recherchée dans le droit divin ${ }^{17}$. En droit français, l'élévation de la propriété privée au rang de «droit inviolable et sacré » par la Déclaration de 1789, est rendue possible grâce à la conjonction du divin et du profane (1.1). La force du sacré va concourir à poser solidement, les bases d'un ordre social, qui se voudra libéral (1.2).

\subsection{De la conjonction du divin et du profane dans la définition juridique du droit de propriété}

Sous l'Ancien Régime, le dominium appartient au roi, autorité de droit divin ${ }^{18}$. La Révolution française, en bouleversant l'ordre qui était établi pendant des siècles, reconsidère le régime juridique général de la propriété. La propriété est envisagée sous un angle juridique différent. Les sujets de droit privé peuvent désormais s'en prévaloir très largement. Le régime juridique est même réorganisé à leur profit. Pour autant, l'invocation du sacré n'est pas exclue dans la conceptualisation laïque du droit de propriété (1.1.1), d'où, nous semble-t-il une certaine élasticité de l'esprit laïc révolutionnaire (1.1.2).

\subsubsection{La marque du sacré dans la conceptualisation laïque du droit de propriété}

La vision moderne du droit de propriété puise ses racines dans la théorie du domaine, développée par les théologiens de la fin du Moyen

16. "Qui appartient à un domaine séparé, inviolable, privilégié par son contact avec la divinité et inspirant crainte et respect ». "Auquel on doit un respect absolu».

17. Ennio Cortese, «Théologie, droit canonique et droit romain : aux origines du droit savant ", Comptes rendus des séances de l'Académie des inscriptions et belles-lettres, vol. 146, n ${ }^{\circ} 1,2002$, p. 57-74.

18. Ce monopole est toutefois à nuancer si on s'en tient aux thèses développées par les juristes des $\mathrm{XVII}^{\mathrm{e}}$ et $\mathrm{xVIII}^{\mathrm{e}}$ siècles. Sur la distinction entre domaine direct et domaine utile, voir Édith Géraud-Llorca, "La doctrine et la propriété à la fin de l'Ancien Régime. 1750-1789", disponible sur http://www.upicardie.fr/labo/curapp/revues/root/27/geraudllorca.pdf (consulté le 24 octobre 2013). 
Âge et du début des Temps modernes ${ }^{19}$. La propriété est le résultat d'un droit naturel de l'homme sur les choses qui l'entourent. Cette manière de voir est empruntée à la construction des anciens penseurs de l'Église.

"L'idée que le domaine originairement détenu par l'homme est lui aussi en son essence un droit, c'est-à-dire une puissance dont il est illégitime d'entraver le développement [...] semble donc fondamentalement constituer la conséquence du lien établi par la pensée théologique entre le domaine divin et le domaine humain. C'est parce que le domaine de l'homme participe du domaine de Dieu ou bien, pour certains, en prolonge l'action sur terre, le représente, qu'il constitue lui aussi, comme le domaine divin, un jus qui s'impose à tous ${ }^{20}$.»

Le droit de propriété perçu comme un droit naturel de l'homme peut être expliquée à partir de la pensée des jus naturalistes, dont l'un des éminents penseurs est John Locke (1632-1704). Celui décrit l'état de nature comme un lieu paradisiaque, à l'instar du jardin d'Éden, avant la survenue du péché originel. Dans la philosophie juridique lockéenne, le droit de propriété sera associé à toute une série de droits qui doivent être reconnus aux individus : le droit à la vie, à la liberté, à la sécurité, à la liberté de conscience ainsi que le droit à la résistance si les droits précités sont menacés. Les analogies avec certaines dispositions de la Déclaration des droits de l'homme et du citoyen sont frappantes, attestant indéniablement d'une influence inspiratrice de la pensée de Locke sur le droit révolutionnaire français.

Le raisonnement de John Locke sur la propriété procède d'un postulat théologique. La propriété est un droit légitimé par son fondement moral. Ce fondement moral repose sur l'existence d'un Créateur dont la volonté doit servir de commandement à toutes les créatures rationnelles. Ces créatures rationnelles la considèrent comme une loi morale envers laquelle elles doivent se sentir obligées parce que «tous les hommes sont l'œuvre d'un seul Créateur tout puissant et infiniment sage, tous les serviteurs d'un seul Souverain Maître envoyés dans le monde par son propre ordre et pour ses affaires; ils sont sa propriété à lui qui les faits, et qui les a destinés à durer selon son bon plaisir et celui de nul autre ${ }^{21} »$.

19. On distingue le "domaine éminent» du seigneur du "domaine utile " correspondant à la jouissance d'un fonds détaché de la seigneurie. Voir Didier Veillon, "L'évolution de la distinction du domaine direct et du domaine utile en France du $\mathrm{XVI}^{\mathrm{e}}$ au XVIII ${ }^{\mathrm{e}}$ siècle", disponible sur http://droit.univ-poitiers.fr/poitiers-roma/IMG/pdf/VEILLON_L_evolution_de_la_distinction_du_ (consulté le 24 octobre 2013).

20. Marie-France Renoux-Zagamé, op. cit., p. 39.

21. John Locke, Second Traité du gouvernement civil, $₫ 6$. 
Locke soutient que, même dans l'état de nature, les individus sont propriétaires d'eux-mêmes et de leurs actions tant qu'ils ne violent pas le principe fondamental de la loi naturelle. «La loi fondamentale de la nature s'identifie à la conservation de l'humanitée ${ }^{2}$.» Comme le docteur de l'église catholique saint Thomas d'Aquin en son temps, Locke défend le principe de redistribution des surplus de propriété à l'égard de ceux se trouvant dans un état de nécessité. C'est la prescription des devoirs envers son prochain, développée par les jus naturalistes dans leur vision $\mathrm{du}$ «droit social privé ${ }^{23} »$.

C'est le principe mutualisé du droit de conservation qui doit toutefois s'accommoder du total respect de la propriété individuelle. Ce droit étant fondé dans la nature, justifie que tout individu qui entre en société pourvu de sa propriété doit bénéficier de la protection qui convient par le pouvoir normatif, en faisant prendre en compte cette protection par la loi civile. L'objectif consiste également en la protection des futurs propriétaires. Le droit révolutionnaire prend le même parti en inscrivant dans la Déclaration des droits de l'homme la haute protection du droit de propriété en l'érigeant en droit sacré.

\subsubsection{L'univers juridique révolutionnaire caractérisé par l'élasticité de l'esprit laïc}

L'avènement de la République marque le net recul de l'emprise de l'Église catholique sur la société française de l'époque. La préférence pour une vision laïque de la société l'emporte très nettement si on s'en tient aux dispositions de la Déclaration des droits de l'homme et du citoyen qui dispose de la liberté de conscience et de culte. Au sens large, la laïcité renvoie notamment à une perte d'emprise de la religion sur la société. Synonyme de sécularisation, ce processus est plutôt progressif que brutal, et se vérifie également pour la France. Certains auteurs allant jusqu'à affirmer que "la République laïque et l'Église catholique se sont faits l'une à l'autre ${ }^{24} »$. La Révolution française accentue pourtant une rupture fondamentale dans l'histoire des rapports entre religion et pouvoir politique. En dissociant l'une de l'autre, elle

22. Ibid., $\$ 135$.

23. Voir Theodorus Meyer, Institutiones juris naturalis, seu philosophiae moralis universae, Fribourg, Herder, 1900, p. 1-89.

24. Émile Poulat, Documents Episcopat, bulletin du Secrétariat de la conférence des évêques de France, $n^{\circ}$ 15, octobre 1990. Cité dans Conseil d'État, Rapport public 2004. Jurisprudence et avis de 2003. Un siècle de laïcité, Paris, La Documentation française, «Études et Documents», p. 246. 
remet en cause l'Ancien Régime. L'Ancien Régime de gouvernement de la France correspond à la coïncidence entre Nation et religion. Au caractère confessionnel de l'État, il faut y ajouter le rôle social proéminent de l'Église catholique ainsi que la responsabilité qui lui incombe, dans les actes de la vie quotidienne, en particulier l'état civil, l'enseignement, les soins hospitaliers.

L'État civil séculier est institué dès 1792, introduisant un distinguo inconnu jusque-là entre la religion et la société pour les événements clés de la vie : la naissance, le mariage et le décès.

Le droit positif devient la norme tandis que la régulation théocratique et le jus naturalis deviennent l'exception. Toutefois, la critique, par la philosophie des Lumières des dogmes catholiques n'a pas raison du sacré, si on se réfère à la disposition de la Déclaration des droits de l'homme et du citoyen consacrée à la propriété qualifiée de "sacrée ». En effet, les Révolutionnaires n'établissent pas une rupture nette entre le pouvoir temporel et le pouvoir spirituel. Le premier cherchant en permanence à contrôler le second sans envisager son extinction. Tout en soulignant l'intermède de la déchristianisation, le développement du culte de l'Être suprême et surtout l'incise de la constitution de l'an III qui préfigure la loi de 1905, le Concordat pour sa part va tout simplement constater que «la religion catholique est la religion de la grande majorité des français ". Il faut se rendre à l'évidence, une Révolution aussi brutale et fulgurante qu'elle peut être ne parvient pas à faire disparaître des caractères qui ont structurés la société pendant des siècles. Aussi parle-t-on avec la Constitution civile du clergé, en 1790, condamnée par le pape Pie VI de "véritable religion révolutionnaire ${ }^{25} »$. Le pouvoir spirituel ne saurait cependant guider le nouvel ordre révolutionnaire mis en place. Concomitamment, les nouveaux maîtres du pouvoir de l'État prennent conscience de l'intérêt de développer une vision utilitariste de la religion. Il n'est pas question d'un rejet pur et simple du sacré. Loin d'une opposition farouche entre normes légales et normes religieuses, les dispositions de l'un et l'autre système peuvent se compléter. La Déclaration des droits de l'homme et du citoyen, qui est encore aujourd'hui, l'une des composantes du bloc de constitutionnalité ${ }^{26}$, n'est ni l'équivalent d'un renoncement aux origines

25. Ibid., p. 250.

26. Voir Georges Vedel, «La place de la Déclaration de 1789 dans le bloc de constitutionnalité », dans Conseil constitutionnel, La Déclaration des droits de l'homme et du citoyen et la jurisprudence, colloque des 25 et 26 mai 1989 au Conseil constitutionnel, Paris, PUF, 1989, p. 49 et suiv. et Louis Favoreu, «Bloc de constitutionnalité», dans 
chrétiennes, ni à la part d'identité chrétienne de la France. Le cadre laïc fixé par la loi repose, en effet, sur deux grands principes: la liberté de conscience et le principe de séparation. Le sacré n’est pas éradiqué mais réorganisé.

La place occupée par la liberté et du droit de la propriété privée concourt à la mise en place d'un nouvel ordre économique qui révèle le caractère libéral de la Révolution française. Le caractère sacré du droit de propriété est ainsi clairement mis en exergue pour parvenir au plein avènement de l'ordre économique libéral.

\subsection{De l'utilitarisme du sacré comme fondant un ordre économique libéral}

Le recours au sacré poursuit un objectif précis. Celui de convaincre les sujets de droit, du bien-fondé de la norme posée. C'est une approche habile et pragmatique des choses. Au lendemain de la Révolution française, il faut rappeler que les nouveaux citoyens français sont porteurs d'une identité personnelle et collective, fortement marquée par la foi chrétienne et la religion catholique. Le recours au sacré est mis au service de l'établissement d'un ordre social libéral (1.1.1) au sein duquel émerge une protection particulièrement accrue du droit de propriété (1.1.2).

\subsubsection{L'empreinte libérale de la Révolution française}

Les chantres renommés du droit naturel, tels que John Locke et JeanJacques Rousseau considèrent que l'exercice des droits politiques sert de catalyseur au droit à la liberté et à la propriété de soi-même. L'individu va échapper à la logique du système collectif qui profite plutôt, sous le règne de l'Ancien Régime, à l'arbitraire du souverain. "Au prix d'un long travail de va-et-vient entre éclaircissements d'ordre conceptuel et démentis opposés par les faits, les révolutionnaires devaient arriver à concevoir la notion d'un droit de propriété privée suffisant pour fonder une économie libérale de marché ${ }^{27}$.»

Le décret du 20 août 1792 dispose : «l'Assemblée nationale, considérant que l'affranchissement des propriétés, en assurant l'indépendance absolue des citoyens, peut seul leur procurer la jouissance pleine et

Olivier Duhamel et Yves Mény (dir.), Dictionnaire constitutionnel, Paris, PUF, 1992, p. 87 et suiv.

27. Colette Capitan, «Propriété privée et individu-sujet-de-droits. La genèse historique de la notion de citoyenneté", L'Homme, $\mathrm{n}^{\circ}$ 153, 2000, p. 65. 
entière de la liberté...». À la suite de la Constitution du 3 septembre 1791, cet acte normatif considère aussi la propriété comme une condition de la liberté. Prenant le contre-pied de l'Ancien Régime, la terre n'est plus perçue comme l'emblème de l'asservissement, mais au contraire le signe de la liberté.

La propriété est la manifestation éclatante de l'affirmation de l'identité individuelle et de l'exercice des droits juridiques et des droits politiques. Les constitutions révolutionnaires successives s'appuient sur la théorie de la conservation, et déclarent ainsi : "l'homme libre est celui qui ne dépend d'aucun autre, qui n'appartient à personne.» Cette disposition est la traduction de l'évangile exprimée par saint Augustin demandant aux hommes de n'attribuer le titre de maître à personne, car «le seul Maître de tous est au $\operatorname{ciel}^{28}$ ». Étant le propriétaire de toute chose, le Maître du ciel peut ainsi faire aux hommes don de la terre pour en tirer la subsistance indispensable à leur vie. Il contribue ainsi de manière décisive à la conservation de l'espèce humaine. Il est donc normal que la propriété privée soit sacralisée.

Cette sacralisation du droit de propriété privée par le pouvoir normatif révolutionnaire, s'explique aussi par le fait que pour garantir sa propre conservation l'homme a besoin d'appliquer son travail à quelque chose. L'homme est libre, s'il s'inscrit dans un cycle de production pour luimême. La propriété privée révolutionnaire est le fruit de l'industrie, c'est-à-dire du travail. L'émancipation de la Nation symbolisée par le territoire et le nouveau système politique, fait de cette dernière l'entité désignée, héritière de l'ancienne classe dominante, pour garantir «souverainement » propriété privée et libertés individuelles.

L'ordre libéral institué par la Révolution repose donc sur la canonisation du droit de propriété privée, classé par l'article 2 de la Déclaration des droits de l'homme et du citoyen de 1789 parmi « les droits naturels et imprescriptibles de l'homme », au même titre que la liberté, la sûreté et la résistance à l'oppression. Mais, la liberté et la propriété indissolublement liées dans l'individualisme libéral sont les deux grands droits naturels révolutionnaires.

L'ordre libéral révolutionnaire envisage la possibilité d'atteinte au droit de propriété, mais non sans avoir rappelé à l'article 17 de la Déclaration des droits de l'homme et du Citoyen son caractère «inviolable et sacré ». Cette atteinte peut intervenir si et seulement si «la 
nécessité publique, légalement constatée, l'exige » évidemment, et "sous la condition d'une juste et préalable indemnitée ${ }^{29}$ ".

Napoléon Bonaparte ne va pas procéder à la dénaturation de la philosophie libérale qui préside au régime juridique du droit de propriété. Il léguera à la postérité cette déclaration lors de la préparation du Code civil : « la propriété c'est l'inviolabilité dans la personne de celui qui la possède ${ }^{30}$.»

\subsubsection{Le droit de propriété privée : l'intangibilité d'un droit?}

Il n'y a pas de césure entre l'esprit qui domine le régime juridique du droit de propriété voulu par les Révolutionnaires et celui qui préside lors de la confection du Code civil. Le régime juridique en droit civil obéit à la logique de la Déclaration des droits de l'homme et du citoyen du 26 août 1789 qui énonce clairement dans son article 17 que : «la propriété étant un droit inviolable et sacré, nul ne peut en être privé...»

Sous le règne de l'Ancien Régime, le territoire de la France était la propriété du Roi, qui en disposait selon son bon vouloir. La Révolution française change la donne en faisant muter cette conception de la propriété. L'un des illustres artisans du Code civil, Portalis, se fait l'écho de la critique de l'Ancien Régime en déclarant que: "dans les États despotiques, où le prince est propriétaire de tout le territoire, où tout le commerce se fait au nom du chef de l'État et à son profit, il y a plus de juges et de bourreaux que de lois ${ }^{31}$.»

En ne dédisant pas la sacralisation du droit de propriété dont on connaît les racines théologiques, le Code civil de 1804 conforte l'idéologie libérale tout en libérant un droit des contrats extrêmement favorables aux propriétaires. Le contrat, est en effet, en général, un instrument de la propriété. C'est par le truchement du contrat que l'on use du droit de propriété par la vente, la location ou la succession. Le droit des contrats dérivé du droit de propriété œuvre en faveur des propriétaires en offrant une théorie générale fondée sur la reconnaissance de certains droits naturels. L'article 544 du Code civil est ainsi tout d'abord libellé: «la

29. Il faut tout de même préciser que l'expropriation motivée par la necessitas n'est pas absente sous l'ère monarchique. Dès le $\mathrm{XIV}^{\mathrm{e}}$ siècle, le pouvoir royal manifeste cette intention. Voir Jean-Louis Mestre, «L'expropriation face à la propriété (du Moyen Âge au Code civil)», Droits, 1985, p. 51-62.

30. Cité par François Terré, «L'évolution du droit de propriété depuis le Code civil », Droits, $\mathrm{n}^{\circ} 1,1985$, p. 2.

31. Jean-Étienne-Marie Portalis, Discours préliminaire au premier projet de Code civil, 1801, p. 15. 
propriété est le droit de jouir et de disposer des choses de la manière la plus absolue.»Ce premier membre de phrase, singulièrement la mention "de la manière la plus absolue", rappelle indubitablement la formule de la Déclaration des droits de l'homme et du citoyen, c'est-à-dire le caractère "inviolable et sacré » du droit de propriété. La suite de la disposition pourrait laisser croire que ce droit est vidé de son sens en précisant «... pourvu qu'on en fasse pas un usage prohibé par les lois et les règlements». Il n'en est rien.

Il faut comprendre que l'ordre public, en agissant comme rempart, peut dans des circonstances bien particulières, primer sur la liberté individuelle, en vue de protéger la société. L'arbitraire et les abus pouvant être aussi l'œuvre des personnes physiques et morales de droit privé et non pas simplement du pouvoir étatique. L'abus de droit présente un visage multiple ${ }^{32}$. La stabilité sociale effective, si chère à Maurice Hauriou $^{33}$, est d'ailleurs l'une des conditions d'efficience du Code civil. Portalis, le principal des quatre rédacteurs du Code civil, a livré la définition suivante: "un corps de lois destinées à diriger et à fixer les relations de sociabilité, de famille et d'intérêt qu'ont entre eux les hommes qui appartiennent à la même cité ${ }^{34}$.»

Il est également intéressant de constater que le titre III du Code civil, qui énonce la théorie générale du contrat, se trouve dans le livre III du Code, intitulé: «Des différentes manières dont on acquiert la propriété». Il apparait donc clairement que les codificateurs poursuivaient l'objectif de protection de la propriété et des relations contractuelles. Portalis fournit l'éclairage nécessaire lorsqu'il souligne : «en traitant des contrats, nous avons d'abord développé les principes de droit naturel qui sont applicables à tous, et ce, pour assurer le respect de certains principes fondamentaux tels que la bonne foi, le respect des bonnes mœurs, la liberté de disposer de ses biens ${ }^{35} \ldots »$

Construit autour de la notion de la propriété individuelle, cette vision révolutionnaire et napoléonienne du droit de propriété souffre d'analyse

32. Voir René Dussault, «De l'abus de droit», Les cahiers de droit, vol. 4, $\mathrm{n}^{\circ}$ 3, 1961, p. 114-125.

33. Voir notre contribution sur la question, Pierre-Yves Chicot, "La notion d'ordre social dans la pensée de Maurice Hauriou : contribution à l'étude de son œuvre», RFDA, $\mathrm{n}^{\mathrm{o}} 3$, mai-juin 2009, p. 419-432.

34. Cité par Catherine Delplanque, «Origine, signification et portée du Code civil en France», Association française pour l'histoire de la justice, juillet 2004, p. 2, disponible sur http://www.afhj.fr/ressources/code-civil.pdf (consulté le 25 novembre 2013).

35. Jean-Étienne-Marie Portalis, op. cit., p. 50. 
critique. Celle-ci est décrite par certains juristes comme l'édification par le Code civil «d'un véritable rite» du propriétarisme ${ }^{36}$. Cette critique n'a manifestement pas eu raison de l'affirmation de la propriété privée des moyens de production, malgré la parenthèse, en France, au début des années 1980, d'une ère de nationalisation de biens privés. Du point de vue du droit au logement la critique peut être sévère si on en juge le propos qui suit : « face à la mise en concurrence d'un droit fondamental classique - le droit de propriété - et d'un droit fondé sur le principe du respect de la dignité humaine érigé, en France, en objectif social de valeur constitutionnelle - le droit à un logement décent - le juge national ne devrait-il pas dépasser la querelle théorique de la primauté d'un droit par rapport à l'autre et refuser d'admettre que dans une société démocratique, des locaux d'habitation restent vides du seul bon vouloir de leur propriétaire ${ }^{37}$.»

\section{De la sacralisation moderne du régime de la propriété privée des moyens de production}

De manière générale, les grands systèmes de pensée sociale postulent la revendication de l'épanouissement de l'homme. Dans le régime de la propriété collective des moyens de production, l'État œuvre forcément pour le bien commun des individus. Dans le régime de la propriété privée des moyens de production, la liberté individuelle participe à la création des conditions nécessaires, à l'accomplissement de tous.

Les conceptions s'affrontant, il est possible qu'il soit porté atteinte à la propriété privée par des interventions de la puissance publique. Le sacré cesse alors d'être l'apanage de la propriété privée. Le sacré récusé comme

36. Cité par Thierry Ménissier, "Peut-on rendre incontestable le droit de propriété», disponible sur http://tumultieordini.over-blog.com/6-categorie-10703567.html (consulté le 25 novembre 2013).

37. Pierre Lambert, «Le droit de l'homme à un logement décent », Revue trimestrielle des droits de l'homme, 2001, p. 48-54. La ministre de l'Égalité des territoires et du Logement ( $\mathrm{M}^{\mathrm{me}}$ Cécile Duflot) a formulé la proposition d'un projet de loi sur l'accès au logement et à l'urbanisme rénové. L'exposé des motifs repose sur l'idée principale suivante: "Face à la dégradation des conditions d'accès au logement et l'exacerbation des tensions sur les marchés immobiliers, le présent projet de loi vise à mettre en œuvre une stratégie globale, cohérente et de grande ampleur destinée à réguler les dysfonctionnements du marché, à protéger les propriétaires et les locataires, et à permettre l'accroissement de l'offre de logements dans des conditions respectueuses des équilibres des territoires». Projet de loi disponible sur http://www.assemblee-nationale.fr/14/projets/pl1179.asp (consulté le 25 novembre 2013). Le logement est un bien de première nécessité et le droit à un logement décent est un objectif protégé par la Constitution. 
fondement de la propriété privée, traduit en fait et en droit la victoire de l'imperium sur le dominium ${ }^{38}$. L'inverse peut être aussi vraie, et dans ce cas, c'est le dominium qui a raison de l'imperium. La propriété privée ressurgit car elle est sacrée et s'inscrit dans «l'ordre naturel», au sujet duquel, l'église s'érige en gardienne ${ }^{39}$.

Au final, dans l'histoire récente française, les atteintes publiques au droit de propriété privée se révèlent être sporadiques (2.1). Invoquant à nouveau ses racines tirées du droit naturel, le fait juridique dominant est celui d'une généralisation du système de la propriété privée des moyens de production (2.2).

\subsection{Des atteintes publiques sporadiques au droit de propriété privée}

Avec la Révolution française, la propriété privée est dite de droit naturel. Elle est décrétée indispensable à la condition de l'homme, car chargée de garantir sa conservation, sa survie. L'ordre socio-économique institué par les Révolutionnaires, en raison de la place conférée à la propriété privée des moyens de production, se confond avec un ordre libéral. Le principe de la propriété privée des moyens de production agit comme un principe directeur. Celui-ci, pouvant toutefois être remis en cause (2.2.1). Le substrat constitutionnel de l'ordre économique français, matérialisé en premier lieu par la Déclaration de 1789, limite les atteintes qui peuvent être portées à la propriété privée. La primauté de la liberté économique demeure et continue à fonder l'ordre socio-économique français (2.1.2).

\subsubsection{De la relativité de la propriété privée comme principe directeur de l'ordre socio-économique français}

La puissance d'interpellation des individus par le recours au sacré a contribué à construire un droit protecteur de la propriété privée. L'homme étant aimé de Dieu, celui-ci ne voit pas d'objection à ce qu'il assure sa propre survie en étant propriétaire de biens, qu'il fera fructifier par son travail. Dans l'ordre libéral, l'usus, l'abusus et le fructus devant permettre la prospérité économique individuelle.

Il se trouve que cette conception d'essence divine du droit de propriété ne reçoit pas un assentiment unanime, et donne lieu à contestation. Sur

38. Guillaume Leyte, "Prescriptibilité et imprescriptibilité dans l'ancien droit public français (xvI ${ }^{\mathrm{e}}$-XVIII ${ }^{\mathrm{e}}$ siècle) », Droits, 2000, p. 31 et suiv.

39. Gérard Filion, «Le capitalisme est athée », Le Devoir, 16 juillet 1949, p. 4. 
le plan du raisonnement théologique, des courants de pensée réfutent la propriété étant comme étant de droit divin ${ }^{40}$.

L'argumentaire consiste moins à nier la légitimité du droit de propriété privée qu'à rappeler le cas de figure toujours possible de la destination universelle des ressources et des biens. Le distinguo est établi entre la propriété privée et l'appropriation qui est la forme pervertie du droit de propriété privée.

Dénonçant ce qui est qualifié de "paganisme propriétaire », l'exercice de ce droit de propriété privée correspond à une démarche antithéologique par le fait qu'elle habille «Dieu avec les attributs de la propriété ». On en arrive à une critique radicale de l'accumulation et de la thésaurisation. Le doute pèse alors sur les possédants si on s'en tient à la parole biblique contenue dans l'évangile de Luc (chapitre XII, verset 15): «Voyez! dit Jésus, Gardez-vous de toute cupidité: si quelqu'un a du surplus, sa vie n'est pas tirée de ses biens.»

Inspiré ou non par la parole divine, le droit n'est jamais irrémédiablement indélébile. Celui-ci est subordonné à la volonté du pouvoir politique en place qui possède la capacité de le maintenir ou de le défaire. Il en va aussi, ainsi, du droit de propriété exercé par les particuliers. On sait que la doctrine de Locke légitimait l'appropriation initiale par le travail, considérée comme le bien propre initial de chacun ${ }^{41}$. Ce bien pouvant toujours faire l'objet d'aliénation par la vente à un tiers ${ }^{42}$, ou par une nationalisation de la part de la puissance publique.

Certains auteurs, à l'image du jurisconsulte Jean-Baptiste-Victor Proudhon, interprètent le droit de propriété comme le fondement de la capacité sociale supérieure qui détermine l'accès à l'autorité politique pour gouverner la cité. Les fonctions publiques, dit-il, « doivent naturellement être remplies avec plus d'assiduité et de zèle par ceux qui peuvent s'y dévouer entièrement et y consacrer tout leur temps : ce sont les propriétaires ${ }^{43} »$.

Cette tendance à l'absolutisation de l'appropriation individuelle conduit à la consécration de l'ordre social des classes possédantes. La proposition de rupture est celle entre autre introduite par le procédé

40. Bernard Van Meenen, op. cit.

41. Sur la vision de Jean-Jacques Rousseau concernant le lien entre travail et propriété, voir Mikhail Xifaras, «La destination politique de la propriété chez Jean-Jacques Rousseau », dans Les études philosophiques, $\mathrm{n}^{\circ}$ 3, 2003, p. 331-370.

42. John Locke, op. cit., chapitre v, $\$ 40-41$.

43. Jean-Baptiste-Victor Proudhon, Traité du domaine de propriété ou de la distinction des biens, considérés principalement par rapport au domaine privé, Dijon, Lagier, 1839 , p. 53. 
$\mathrm{du}$ « renversement de l'individualisme possessif ${ }^{44} »$. Il est question de rénovation de la propriété privée qui doit être entendue à partir d'une dépossession qui interviendra par l'action du pouvoir politique. La propriété privée apparaît progressivement non plus comme la règle mais l'exception, précisément parce que son statut juridique est discuté.

"Au-delà du fondement en nature du droit de propriété, il importe de savoir si la propriété constitue un droit individuel ou un droit social réglé et limité par la loi : l'autorité politique n'est-elle légitime que dans la mesure où elle permet aux individus de conserver leurs biens acquis ou est-elle dotée d'un droit de regard sur l'acquisition, la conservation et la transmission des biens? Le pouvoir peut-il s'ériger en tuteur de la société et décider souverainement de ce qui est légitimement possédé par chacun ${ }^{45}$ ?» La question est celle de la prévalence du dominium des sujets ou de l'imperium du prince.

Montesquieu s'éloignant à cet égard du droit naturel qui fonde la propriété dans la conception lockéenne, estime qu'en démocratie «les richesses donnent une puissance dont un citoyen ne peut pas user pour lui », elle "procure les délices dont il ne doit pas jouir ${ }^{46}$ ». Montesquieu ne suit pas Locke dans sa conception du développement de la société à partir du droit naturel à la propriété ${ }^{47}$. Celui-ci ne plaide pas toutefois contre la sacralité de la propriété privée dans la mesure où d'après ce qu'il rapporte "le bien public est toujours que chacun conserve invariablement la propriété que lui donnent les lois civiles ${ }^{48} »$. La loi civile est ainsi le palladium de la propriété.

Chez Jean-Jacques Rousseau, l'imperium semble l'emporter sur le dominium sans pour autant contester dans le fond la sacralité de la propriété privée. On se rapproche ainsi de la philosophie politique et économique qui légitime l'appropriation publique des moyens de production. L'auteur du Contrat social soutient dans sa théorie républicaine de la propriété «quoique inviolable et sacré, la propriété privée reste soumise à la loi et aux réquisits de la communauté, qui conserve un droit éminent sur elle : [...] le droit que chacun a sur son propre fond est

44. Étienne Balibar, La Proposition de l'égaliberté, Paris, PUF, «Actuel marx confrontation ", 2010, notamment p. 91 et suiv.

45. Blaise Bachofen (dir.), Le Libéralisme au miroir du droit. L'État, la personne, la propriété, ENS Lyon, 2008, p. 98.

46. Montesquieu, De l'esprit des lois, V, 6 .

47. Voir l'étude de Céline Spector, "Variations de la propriété : Montesquieu contre l'individualisme possessif», dans Inventions et critiques du libéralisme. Le pouvoir, la personne, la propriété, Blaise Bachofen (éd.), Lyon, ENS, «Theoria», 2008, p. 95-116.

48. Montesquieu, De l'esprit des lois, XXVI, 15. 
toujours subordonné au droit que la communauté a sur tous, sans quoi il n'y aurait ni solidité dans le lien social, ni force réelle dans l'exercice de la souverainetét ${ }^{49}$ \%.

En France, l'appropriation publique moderne (1982) de la propriété par les nationalisations procède de la participation des travailleurs à la bonne marche de l'outil de production et à un partage plus équilibré des richesses ${ }^{50}$, à la recherche d'une plus grande performance économique de l'État ${ }^{51}$ et d'un choix de société ${ }^{52}$. L'atteinte portée au dominium par la puissance publique est une tentative de désacralisation de la propriété privée. Le juge constitutionnel sollicité pour dire le droit en la matière, plaidera pour une protection de la liberté économique, et par conséquent du droit de propriété.

\subsubsection{De la préservation de la liberté économique comme principe juridique directeur de l'ordre socio-économique français}

Au début des années 1980, à la faveur de l'accession à la magistrature suprême du leader de la gauche socialiste et communiste, François Mitterrand, le gouvernement de Pierre Mauroy entreprend l'extension du secteur public. Celle-ci «s'inscrit en premier lieu dans une logique politique " et se révèle être un "compromis entre les thèses marxistes sur l'appropriation collective des moyens de production et les thèses sociale-démocrates, qui s'inscrivent, au contraire, dans le cadre d'une économie de marché ${ }^{53}$ ".

Cette atteinte portée par l'administration à la propriété privée peut être interprétée comme une forme de désacralisation de celle-ci. Sur le

49. Jean-Jacques Rousseau, Euvres complètes, Paris, Gallimard, «Pléiade », t. III, 1964, p. 367.

50. Voir aussi David Pinkney, «The French Experience in Nationalization: 1944-1950", dans Edward Mead Earle, Modern France, Princeton University Press, 1951, p. 344.

51. Michel Durupty, Les Nationalisations, Paris, Economica, 1982, p. 206.

52. Jean-Pierre Fourcade, Jean Chérioux et Étienne Dailly, Rapport fait au nom de la Commission spéciale chargée d'examiner le projet de loi de nationalisation déposé le 16 novembre 1981, documents du Sénat, rapport $n^{0}$ 56, p. 142-143 et 97-98. Disponible sur http://www.senat.fr/rap/l81-56/l81-56.html (consulté le 25 novembre 2013). Voir également Jean-Charles Asselain, « La dimension sociale des nationalisations de 1982 ", Revue économique, vol. $34, \mathrm{n}^{\circ}$ 3, 1983, p. 536-576.

53. Christian Stoffaes, «Objectifs économiques et critères de gestion du secteur public industriel », Revue économique, vol. 34, $\mathrm{n}^{\circ} 3,1983$, p. 577. 
strict plan du droit, la liberté du commerce et de l'industrie, "composante de la liberté fondamentale d'entreprendre ${ }^{54}$ » est en cause. Dans les conclusions sur la célèbre affaire Daudignac, le commissaire du Gouvernement Gazier affirmait que «là où aucune loi n'est intervenue, le principe subsiste toujours, qui demeure le droit commun de l'activité industrielle en France et que la loi des 2-17 mars 1791 proclamant la liberté du commerce et de l'industrie restait applicable ${ }^{55}$ ».

Le Conseil constitutionnel a lui-même reconnu, au-delà du «libre exercice de l'activité professionnelle ${ }^{56}$ ", «la liberté d'entreprendre ${ }^{57}$ ", en se fondant les législations d'avril et juin 1791. Ces jurisprudences favorables à l'ordre libéral n'ont pas empêché les juges de la rue de Montpensier de considérer que la liberté d'entreprendre, n'est ni générale, ni absolue, et s'exerce par conséquent dans le cadre d'une réglementation instituée par la loi ${ }^{58}$.

Cette limite à la liberté d'entreprendre, est, elle-même limitée. Le juge constitutionnel estime que s'il est « loisible au législateur d'y apporter des limitations exigées par l'intérêt général » ce n'est «qu'à la condition que celles-ci n'aient pas pour conséquence d'en dénaturer la portée ${ }^{59}$ ".

Le Conseil constitutionnel a affirmé la pleine valeur constitutionnelle du droit de propriété privée. S'appuyant sur l'article 2 de la Déclaration des droits de l'homme et du citoyen, et non simplement sur son article 17, le Conseil constitutionnel a reconnu en 1982 le caractère éminent du droit de propriété privée ${ }^{60}$. Celui-ci est ainsi mis sur le même plan que la liberté, la sûreté et la résistance à l'oppression, « au nombre des droits naturels et imprescriptibles de l'Homme dont la conservation constitue l'un des buts de la société politique».

En matière de nationalisation, le Conseil a pu exposer l'énoncé suivant dans son célèbre arrêt du 16 janvier 1982 : «l'article 34 de la Constitution qui place dans le domaine de la loi les nationalisations d'entreprises et qui confie à la loi la détermination des principes fondamentaux du régime de la propriété, ne saurait dispenser le législateur, dans l'exercice

54. CE, 12 novembre 2001, Commune de Montreuil-Bellay, Recueil Lebon, p. 551.

55. CE, 22 juin 1951, Daudignac, Recueil Lebon, p. 362.

56. CC, décision $\mathrm{n}^{0} 76-87 \mathrm{~L}$ du 3 mars 1976, Recueil CC, p. 49.

57. CC, décision $\mathrm{n}^{\mathrm{o}}$ 81-132 DC du 16 janvier 1982, Recueil CC, p. 18.

58. CC, décision $\mathrm{n}^{\circ}$ 85-200 DC du 16 janvier 1986, Recueil CC, p. 6.

59. CC, décision $\mathrm{n}^{0}$ 89-254 DC du 4 juillet 1989, Recueil CC, p. 41.

60. CC, décision $n^{0} 81-132$ DC du 16 janvier 1982, Recueil Dalloz, 1983, p. 169, note L. Hamon ; JCP, 1982, II, 1988, note Nguyen Quoc Vinh et Claude Franck; Gazette du Palais, 1982, 1, p. 67, note Stéphane Piédelièvre et Philippe Dupichot; RCDIP, 1982, p. 349, note Jean-Marc Bischoff. 
de sa compétence, du respect des principes et des règles de valeur constitutionnelle qui s'imposent à tous les organes de l'État. »

Le Conseil constitutionnel, a par ailleurs jugé que la dépossession par la puissance publique est subordonnée à une juste et préalable indemnisation ${ }^{61}$.

Affirmé comme un "principe fondateur» de la démocratie, le droit de propriété privée ne saurait être vidé de son sens par le législateur même en cas d'alternance politique. En combinant reconnaissance constitutionnelle de la liberté d'entreprendre et droit de propriété privée, François Luchaire a pu conclure que le "Conseil relie la propriété à la liberté d'entreprendre; c'est donc un régime économique qui est ainsi affirmé. La France est une République sociale ${ }^{62}$; elle n'est pas une République socialiste ${ }^{63} »$. Le professeur Luchaire reprend certainement à son compte un débat déjà ancien qui a prévalu au $\mathrm{XIX}^{\mathrm{e}}$ siècle lorsqu'il s'agissait de tisser un lien entre droit au travail et droit de propriétét ${ }^{64}$.

On pourra toujours arguer du fait que le droit de propriété privée reste fragile face au droit de préemption dont est titulaire le pouvoir administratif ou face au droit d'expropriation pour cause d'utilité publique dont peut se prévaloir ce même pouvoir ${ }^{65}$. Là aussi, le droit de propriété privée reste dans une certaine mesure protégé, car le propriétaire peut toujours introduire une action pour contester la légalité des actes de l'administration. Le juge pouvant faire droit aux prétentions du propriétaire lésé, en se référant à la Convention européenne de sauvegarde des droits de l'homme ${ }^{66}$. Au surplus, dans le cas de l'expropriation pour cause d'utilité publique, l'exproprié bénéficie de la protection du juge judiciaire, gardien du droit de propriété privée depuis la loi du 8 mars

61. CC, décision $\mathrm{n}^{\circ}$ 81-132 DC du 16 janvier 1982 - Lois de nationalisation, Recueil CC, p. 18.

62. Article $1^{\text {er }}$ de la Constitution de 1958.

63. Cité dans «Le rôle des Cours constitutionnelles dans le processus de réformes économiques: l'expérience du Conseil constitutionnel», disponible sur http://www.conseil-constitutionnel.fr/conseil-constitutionnel/root/bank_mm/pdf (consulté le 25 novembre 2013).

64. Voir Victor Considérant, Théorie du droit de propriété et droit au travail, Paris, Librairie phalanstérienne, 1848.

65. L'État-providence promoteur du droit au logement interviendra tout au long du $\mathrm{xx}^{\mathrm{e}}$ siècle pour protéger les droits des locataires face aux bailleurs. La puissance publique n'est pas pour autant soumise à une obligation de résultat. Voir ENA, direction des études, Le Droit au logement, juillet 2005.

66. CE, 10 mars 2010, Levy c/ Communauté urbaine de Strasbourg, req. $n^{\circ} 323081$. 
1810. Le propriétaire dépossédé possède une autre voie, celle qui consiste à tenter d'obtenir gain de cause auprès du juge européen ${ }^{67}$.

Une partie de la doctrine estime que le droit de propriété privée apparait comme un droit de "second rang" par rapport aux droits et libertés de "premier rang" que constituent la liberté individuelle, la liberté d'association, la liberté de la presse ou la liberté de l'enseignement ${ }^{68}$. Si un tel point de vue est défendu c'est en réaction au fait qu'on a le sentiment que le régime juridique de protection national et européen dont jouit le droit propriété privée gagne significativement du terrain.

\subsection{De la généralisation de la propriété privée des moyens de production}

La propriété privée est de droit naturel. Cet argument fondamental fonde sa sacralité dans l'ordre libéral révolutionnaire. À cette époque, la propriété individuelle est perçue comme la forme la plus aboutie de droit subjectif et la base de l'organisation sociale révolutionnaire. Le but ultime réside dans la recherche de l'harmonie et de la prospérité du corps social.

Le marxisme critiquera avec virulence la propriété individuelle et le subjectivisme libéral, lui préférant un ordre public économique dominé par la socialisation. La transposition pratique, même partielle de cette vision, a été effective en France, au travers d'initiatives prises par la puissance publique, donnant lieu à la nationalisation d'entreprises privées.

Le bouleversement historique qui se produit, à la fin des années 1980, non sans le soutien idéologique de l'Église catholique, contribue en France et ailleurs, en réaction au communisme, à un processus de généralisation de la propriété privée des moyens de production (2.2.1). Le renouveau d'un droit public économique libéral est articulé autour de la propriété privée et de la liberté d'entreprendre (2.2.2).

67. CEDH, 2 juillet 2002, Motais de Narbonne c/ France, AJDA, 24 novembre 2003, p. 2144.

68. Voir François Terré, Les Grands Arrêts de la jurisprudence civile, 12 édition, 2007, p. 11 et suiv. 


\subsubsection{La propriété privée contemporaine soutenue par la philosophie de l'Église catholique}

Il est possible d'établir une relation entre la sphère temporelle de l'économie et la sphère intemporelle de la religion ${ }^{69}$. « Lorsque l'esprit puritain et l'esprit capitaliste se confondent, [...] la réussite en affaire devient le signe de la bénédiction divine ${ }^{70}$.»

L'Église catholique n'oppose pas un refus absolu aux affaires qui ont vocation à croître dans l'univers libéral. Toutefois, elle affirme une position de vives critiques face au capitalisme concentrationnaire à tendance ploutocratique ${ }^{71}$ tel qu'il se révèle être aujourd'hui.

Dans la tradition de l'Église, il s'agit de faire en sorte de combattre «le déséquilibre économique, la possession énorme des biens, le désir bien résolu de la conserver, d'en faire un instrument de domination, un système dans lequel la richesse s'établira finalement comme sur un roc, en vue non seulement de soutenir sièges et assauts, mais encore d'agir et d'assaillir à son tour, jusqu'à la victoire écrasante ${ }^{72}$ ».

Si les prescriptions catholiques ne s'opposent pas aux œuvres sociales et économiques du travail, de l'épargne et du prêt, elles rejettent le libéralisme qui se "fonde sur ces conceptions erronées et s'arroge un droit illimité sur la propriété en dehors de toute subordination au bien commun». L’Église réprouvant alors celui-ci en le qualifiant " comme contraire au droit naturel ${ }^{73}$ ». Cette orientation du libéralisme économique qui nie l'altérité, fait obstacle à la "propriété effective» pour l'ouvrier, tout en condamnant "la petite et moyenne propriété » à l'effritement ${ }^{74}$.

69. André Encrevé et Jean-Marie Mayeur, Les Chrétiens et l'économie, Paris, Centurion, 1991.

70. Frédéric Mauro, «Tawney (R. H.) - La religion et l'essor du capitalisme », Revue économique, vol. $3, \mathrm{n}^{\circ} 6,1952$, p. 885-886.

71. L'encyclique Quadragesimo Anno, mettant à jour la lettre léonienne, dont elle commémorait le $40^{\mathrm{e}}$ anniversaire, s'exprimait ainsi: «Ce qui, à notre époque, frappe tout d'abord le regard, ce n'est pas seulement la concentration des richesses, mais encore l'accumulation d'une énorme puissance, d'un pouvoir économique discrétionnaire, aux mains d'un petit nombre d'hommes qui, d'ordinaire, ne sont pas les propriétaires, mais les simples dépositaires et gérants du capital qu'ils administrent à leur gré ».

72. "L'église catholique et le capitalisme ", disponible sur http://bibnum2.banq.qc.ca/bna/actionnationale/src/1949/07/05/1949-07-05.pdf (consulté le 26 novembre 2013).

73. Déclaration de Pie XII dans l'encyclique à l'occasion du cinquantenaire de l'encyclique Rerum Novarum.

74. Ibid. 
L'ordre public économique libéral défendu par l'Église repose au principal sur la nature sacrée de la propriété privée. Léon XIII, dans la lettre encyclique Rerum novarum sur la condition des ouvriers, datée du 15 mai 1891, nous renseigne abondamment sur la doctrine de l'Église en la matière. L'idée que "toute propriété de biens privés doit être supprimée, que les biens de chacun doivent être communs à tous, et que leur administration doit revenir aux municipalités ou à l'État», est récusée.

La propriété privée est considérée comme s'inscrivant dans l'ordre naturel des choses et vouloir y mettre fin «est souverainement injuste, en ce qu'elle viole les droits légitimes des propriétaires, qu'elle dénature les fonctions de l'État, et tend à bouleverser de fond en comble l'édifice social ». La propriété privée est même décrite comme la finalité légitime d'un parcours de vie car " la raison intrinsèque du travail entrepris par quiconque exerce un métier, le but immédiat, visé par le travailleur, c'est d'acquérir un bien qu'il possédera en propre et comme lui appartenant. S'il met à la disposition d'autrui ses forces et son énergie, ce n'est évidemment que pour obtenir de quoi pourvoir à son entretien et aux besoins de la vie». De ce fait, la philosophie de la propriété collective est présentée comme un obstacle dirimant à l'espoir pour l'ouvrier «d'agrandir son patrimoine».

$\mathrm{La}$ « répudiation » du système de la propriété collective tient en trois points essentiels. Premièrement, le fait que la terre soit donnée en commun aux hommes n'entre pas en contradiction avec la propriété privée. Deuxièmement, l'homme se différencie de l'animal par l'opportunité offerte par le "droit naturel, stable et perpétuel», de posséder. Enfin, les conséquences du système de la propriété collective sont de l'ordre $\mathrm{du}$ « funeste » car "préjudiciables aux ouvriers, contraires aux droits naturels des individus, dénaturant les fonctions de l'État et troublant la tranquillité publique».

La conséquence de l'ensemble de la démonstration est sans appel et sèchement exposée: "qu'il reste donc bien établi que le premier fondement à poser par tous ceux qui veulent sincèrement le bien du peuple, c'est l'inviolabilité de la propriété privée.»

Dans l'acte de posséder et dans la manière d'organiser son régime juridique, le système normatif de la propriété privée proposée par l'Église laisse une large place à la mesure et à l'éthique. Qu'observet-on aujourd'hui ? L'ordre public économique qui a cours, en particulier depuis la fin des années 1980, est aux antipodes d'une telle vision des choses, tant la perception sacrée originelle est devenue fugace. La nature sacrée de la propriété privée tend à servir de prétexte à des objectifs bien 
éloignés du bien commun ${ }^{75}$ et de la conservation de l'homme telle que l'avaient envisagée les révolutionnaires, inspirés par le droit naturel.

\subsubsection{La reconduction d'un droit public économique libéral : la propriété privée et la liberté d'entreprendre}

L'une des grandes figures de l'économie politique libérale française est Jean-Baptiste Say (1767-1832). Dans sa production scientifique ${ }^{76}$, il définit la propriété privée sur des bases qui permettent de placer le propriétaire en position de force. Il indique en effet que «c'est le droit garanti à son propriétaire d'en disposer à sa fantaisie, à l'exclusion de toute autre personne ». La puissance publique joue un rôle fondamental dans la mesure où il lui revient le soin d'en garantir l'exercice. Les usages de la société venant en complément. Cette place offerte au propriétaire est justifiée par «la force corporelle, l'intelligence, les talents naturels qui sont des dons de la nature ». L'abbé Sieyès avait utilisé la formule suivante: "employer ses bras, son industrie et ses capitaux, ainsi qu'il le juge bon et utile à lui-même ${ }^{77}$. " Jean-Baptiste Say distingue une gradation dans la nature sacrée des propriétés. Les facultés industrielles (la prise de risque en entreprenant grâce aux facultés naturelles qui ont été données par la nature) précédant dans le classement qu'il expose, les facultés des capitaux (l'épargne et l'héritage).

Ces considérations économiques libérales ont pénétré notre droit. En droit public économique, la thématique de la liberté constitue un sujet de choix car déterminant essentiel de la propriété privée. On doit au Conseil constitutionnel une lecture de la liberté d'entreprendre jugée plutôt libérale par une partie de la doctrine ${ }^{78}$. Elle est dérivée de l'article 4 de la Déclaration de 1789 : «la liberté consiste à pouvoir faire tout ce qui ne nuit pas à autrui.»

Bien que le Conseil prenne quelque liberté, celui-ci n'a pas eu tort de tirer une telle conclusion en se fondant sur l'esprit révolutionnaire de l'époque et non pas exclusivement sur la lettre. Jean Rivero et Georges Vedel ont soutenu pour leur part que: la «liberté économique » est « implicitement incluse dans la définition de l'article 4 de la Déclaration

75. Zaki Laïdi, Le Sacre du présent, Paris, Flammarion, 2002 ; id., Un monde privé de sens, Paris, Hachette Littérature, 2006.

76. Jean-Baptiste Say, Catéchisme d'économie politique, ou instruction familière qui montre de quelle façon les richesses sont produites, distribuées et consommées dans la société, Paris, Crapelet, 1815, p. 78 et suiv.

77. Législation française ou recueil des Constitutions françaises, Paris, 1826, p. 63.

78. Robert Savy, «La constitution des juges», Recueil Dalloz, 1983, p. 105-110. 
de 1789 "; que les constituants de 1946 "en maintenant la définition traditionnelle de la liberté [...] ont implicitement maintenu cette part de liberté qui en découle nécessairement et sans laquelle la liberté de l'individu, dans un monde où les emplois seraient imposés par voie d'autorité, ne seraient plus qu'un leurre ${ }^{79}$ ».

La confrontation entre ce qui est imposé par l'autorité publique, notamment en usant de la loi, et la liberté de la prise de risque en investissant et en acquérant, participe d'un mouvement de flux et de reflux récurrent ${ }^{80}$, mettant en lumière une "hésitation entre le libéralisme et le socialisme ${ }^{81}$ ", pour reprendre la formule du professeur Pelloux.

Le rapport entre la libre entreprise et des services publics nationaux et locaux devant être gérés par des personnes publiques, masque difficilement la question du statut de la personne qui doit exercer la propriété sur les services publics. Qui doit en être le propriétaire? Est-ce la collectivité publique? Est-ce le particulier (le marché) ? Une autre question surgit tout aussi tôt : l'État peut-il être un entrepreneur?

$\mathrm{Au}$ fond, c'est le choix de l'ordre public économique qui est mis en avant. La crise financière qui sévit depuis 2008, entraînant des répercussions sur le champ économique et social a laissé croire fugacement à un retour de l'État régulateur et interventionniste. Si ce cas de figure n'est pas dans l'absolu à exclure, la tendance actuelle demeure gouvernée par un postulat libéral en vertu duquel: toute intervention de l'État susceptible de remettre en cause les prérogatives de la libre entreprise (ou libre force du marché) et de l'épanouissement de la propriété privée constitue une violation de frontière devant être sanctionnée, car en disharmonie "profonde avec la conception de la société des Révolutionnaires de $1789^{82}$ ».

79. "Les principes économiques et sociaux de la Constitution», Collection droit social, vol. XXX, 1947, p. 29. Voir également Jean-Louis Mestre, "Le Conseil constitutionnel, la liberté d'entreprendre et le droit de propriété », Recueil Dalloz, 1984, p. 1.

80. Le débat sur les 35 heures constitue un exemple récent.

81. Robert Pelloux, «Le préambule de la constitution du 27 octobre 1946 », RDP, 1947, p. 398.

82. Jean-Pierre Théron, "La liberté d'entreprendre: limite à l'intervention publique", dans Propriété et Révolution, actes du colloque de Toulouse 12-14 octobre 1989, Paris, CNRS, 1990, p. 215. 


\section{Conclusion}

À propos du droit de la propriété privée et de la liberté d'entreprendre, on observe que la doctrine se répartit, sur le front des idées, autour de deux conceptions principales. Une doctrine qui constate, non sans une certaine amertume, que la propriété privée n'est plus un droit-liberté mais un droit de créance, car le «Conseil n'accorde au droit de propriété qu'une protection assez modeste ${ }^{83}$ ».

Une autre, qui rend compte cette fois, avec un dépit qui semble flagrant, que le principe de la liberté d'entreprendre et la propriété privée peuvent bénéficier de la protection du juge constitutionnel alors que «le principe de la nécessaire nationalisation des biens et entreprises ayant un caractère de service public ne dispose pas d'une telle protection, dans la mesure où ce juge ne dispose pas du pouvoir d'obliger le législateur à agir. Avec le droit existant, le juge constitutionnel peut empêcher une opération de nationalisation mais il ne peut imposer la réalisation d'une telle opération ${ }^{84}$ ».

L'épreuve de force entre l'esprit du préambule de la Constitution du 27 octobre 1946 et la Déclaration des droits de l'homme et du citoyen du 26 août 1789 est susceptible de trouver un règlement hors de l'espace national. Le droit communautaire manifeste la volonté de maintenir la nature sacrée de la propriété privée, si on s'en tient au raisonnement du juge communautaire, ardent défenseur d'une construction communautaire d'inspiration libérale. En même temps, ce dernier admet que l'intérêt général soit un justificatif aux restrictions qui peuvent être apportées au droit de la propriété privée. Néanmoins in fine, ces restrictions ne doivent pas constituer, " au regard du but poursuivi, une intervention démesurée et intolérable qui porterait atteinte à la substance du droit même ainsi garanti ${ }^{85}$ ». La nature du sacré du droit de propriété reste sauve!

Maître de conférences de droit public université des Antilles et de la Guyane

83. François Terré, op. cit., p. 19.

84. Gérard Quiot, "Service public national et liberté d'entreprendre ", dans CURRAP (dir.), Le Préambule de la Constitution de 1946: antinomies juridiques et contradictions politiques, Paris, PUF, 1996, p. 207 et suiv.

85. CJCE, 22 octobre 1991, aff. C. 44/89, Recueil CJCE n ${ }^{\circ}$ 18-91, p. 2. 